\title{
"MUSICAL EXERCISE" FOR PEOPLE WITH VISUAL IMPAIRMENTS: A PRELIMINARY STUDY WITH THE BLINDFOLDED
}

\author{
Ridwan Ahmed Khan, Myounghoon Jeon
}

\author{
Mind Music Machine Lab, \\ Michigan Technological University, \\ 1400 Townsend Dr., Houghton, MI, USA \\ \{ridwank, mjeon\}@mtu.edu
}

\begin{abstract}
Performing independent physical exercise is critical to maintain one's good health, but it is specifically hard for people with visual impairments. To address this problem, we have developed a Musical Exercise platform for people with visual impairments so that they can perform exercise in a good form consistently. We designed six different conditions, including blindfolded or visual without audio conditions, and blindfolded or visual with two different types of audio feedback (continuous vs. discrete) conditions. Eighteen sighted participants participated in the experiment, by doing two exercises - squat and wall sit with all six conditions. The results show that Musical Exercise is a usable exercise assistance system without any adverse effect on exercise completion time or perceived workload. Also, the results show that with a specific sound design (i.e., discrete), participants in the blindfolded condition can do exercise as consistently as participants in the non-blindfolded condition. This implies that not all sounds equally work and thus, care is required to refine auditory displays. Potentials and limitations of Musical Exercise and future works are discussed with the results.
\end{abstract}

\section{INTRODUCTION}

Physical activity or exercise is necessary for maintaining good health physically [8] and mentally [24]. However, accessibility to exercise is a critical issue for population with disabilities [19, 30]. For example, people with visual impairments suffer from health issues due to lack of exercise [25] [2] [12]. They need active guidance from another person to help them learn and practice the exercise [25]. Even if they have learned the exercise with the help of another person, they may have to do it by themselves sometimes. In such a case, guidance or at least a feedback system telling them if they are doing the exercise correctly can help improve their exercise. Technological interventions can assist people with visual impairments doing the exercise [23]. However, designing an intuitive feedback system to ensure that the user does the exercise correctly and consistently is a difficult goal to achieve. The feedback system should be intuitive and realtime so that people with visual impairments can easily understand how they are doing while exercising. Also, the feedback system needs to be non-visual but clear enough at the Attribution - Non Commercial 4.0 International License.

The full terms of the License are available at

http://creativecommons.org/licenses/by-nc/4.0/

\author{
Tejin Yoon
}

\author{
Neuromechanics Lab, \\ Michigan Technological University \\ 1400 Townsend Dr., Houghton, MI, USA \\ tyoon@mtu.edu
}

same time. The feedback system also needs to be enjoyable so that people with visual impairments get motivated sufficiently. To address these issues, we have designed and evaluated the Musical Exercise system. It is a non-wearable exercise detection system using Microsoft Kinect which uses sound as real-time feedback. The Microsoft Kinect device is placed in front of the user and it tracks the movement of the user's joints. Musical Exercise then analyzes the movement data and decides whether it is a valid exercise. At the same time, Musical Exercise provides sound feedback which conveys the information of how the user is doing in the exercise. For the first implementation, we have selected two exercises - squat and wall sit for the experiment and designed two different audio profiles - continuous sound and discrete sound to test the Musical Exercise system.

In the following sections, after providing related work, we described the design of the Musical Exercise system. Then, we detailed an experiment of the system with 18 sighted participants. The results of the experiment show that Musical Exercise can have potential benefits to people with visual impairments with a proper sound design. The results also indicate that sighted people can also benefit from the Musical Exercise system with intuitive non-obtrusive sound feedback.

\section{HEALTH \& EXERCISE TECHNOLOGY}

There are a huge number of fitness applications [18] that have immense benefits for behavior modification [11], older adults [9, 27], and chronic diseases [29]. Microsoft Kinect is one of the most frequently used devices. It tracks users' hand movements [4], sit posture [28], or running [5], and provides feedback or corrects the exercise form [6]. In a similar line, Kinect or Nintendo Wii has been used in a game for exercise of people with disabilities. Research has been conducted for motor rehabilitation exercise of patients having Parkinson's disease [1, 22], for full body motion of wheel chairs users [10], rehabilitation of patients' movement disorders [17], and upper extremities rehabilitation [13].

In this line of research, multimodal and auditory displays have been an important component. Newbold et al. [21] designed a mechanism by which users can be musically informed of their position in the stretching exercise. This musical information intuitively prevents the users from over stretching. Another study [14] showed that multimodal feedback can be helpful to older adults for performance gain while doing an activity. A system with auditory feedback was also developed by Singh et al. [26] to support exercise for people with chronic pain. The researchers found that when the audio was tailored to one's personality, exercise became psychologically motivating. 


\section{TECHNOLOGICAL ASSISTANCE FOR EXERCISE OF PEOPLE WITH VISUAL IMPAIRMENTS}

Assistive technologies can also help people with visual impairments do exercise or physical activity easily. One such a project is Gobi [20], which helps swimmers with visual impairments to stay on the right way while swimming. It is a wearable device on the thigh which tracks the swimming lane with a camera and guides the swimmers to swim left or right if they are swimming away. Winoto and Tang [31] developed a helmet attaching Arduino and speakers for people with visual impairments to play games or move around. Once the users wear the helmet which has five directional speakers, the system generates the guidance using sound feedback via one of the speakers and haptic feedback as vibration via a mobile phone. Al Zayer et al. [32] explored the idea of using an aerial quadrotor to guide a blind runner in running. Morelli et al. [19] designed an exercise game for people with visual impairments which is similar to a popular tennis game for sighted people. Tactile display was used as their primary feedback, while auditory display was used as the secondary feedback. Rector et al. [23] used a Kinect device with auditory feedback to help people with visual impairments doing yoga exercise. Their system tracks users' body and limb positions and provides speech instructions for a specific yoga pose.

As shown above, researchers have accommodated different feedback systems for people with visual impairments. Tactile or haptic feedback systems are common, but using this type of feedback system comes with some caveats, e.g., the tactile or haptic device needs to be placed on users' body which seems obtrusive. Sometimes, it may distract users because they have physical responses from these devices. On the other hand, some researchers used auditory feedback to convey information about the activity. It is a non-obtrusive approach and users can hear the instructions and follow. However, speech instructions can be limited in number as these instructions are pre-recorded and not in real-time and may not meet the requirement of instructing every possible case the users can face themselves in. Also, speech provides abstract information; e.g., "move your right hand". The distance to move the right hand may vary for different users. Therefore, instructional speech may not cater to the specific need of a specific user. Musical Exercise aims to address these issues with non-speech sounds or musical tones. The benefit of using non-speech is that it can cater to the need of specific users. Users learn to hear the collection of musical notes of a proper form of the exercise. When they are deviating from the proper form, instantly the notes sound different. The sound of one user's proper form may differentiate from another user. In that way, Musical Exercise addresses the issue of giving individualized feedback elaborating non-speech sounds which are not confined by speech sounds.

\section{MUSICAL EXERCISE FOR PEOPLE WITH VISUAL IMPAIRMENTS}

\subsection{Design Goals}

To help people with visual impairments do the exercise in a more comfortable and effective way, we designed and developed Musical Exercise. Below is the list of main design goals:

a) Motivate to do exercise: Musical Exercise was designed to motivate people to keep people regularly do exercise. With this system, people listen to the music of their body movement. Music can create positive emotions and affect in this situation [16]. Thus, by doing exercise which creates the music, people may become more interactive and engaged to the task. An engaging and interactive task creates motivation in people.

b) Learn exercise: Another design goal of Musical Exercise is to make it simple and easily accessible for people with visual impairments to learn different exercises. Once people are introduced to the system and the exercises, they can start learning about those exercises by themselves. They can simulate the sound of the proper form of each exercise.

c) Guide to do exercise: After learning the exercise using Musical Exercise, people can continue using the system as their guidance system while doing exercise. The sound intuitively guides them to maintain the proper form of the exercise and do the exercise in a correct and effective way.

d) Perform the exercise as good as a sighted person: One of the motivations behind Musical Exercise was to enable people with visual impairments to do the exercise as good as sighted people can do. Musical Exercise utilizes Microsoft Kinect to track motions of the user and then uses the tracking data to assist the user perform efficiently through sound feedback. Sound or music is intuitive feedback and instigates more physical responses from the user [7]. Also, sound is nonunobtrusive feedback for people with visual impairments. Therefore, people with visual impairments can do exercise as well as sighted people using the Musical Exercise system.

e) Rely on assistive technology at home: People with visual impairments do not need to go to the gym or take much help from other people. They can use the Musical Exercise system and do exercise with less assistance. More importantly, they can use Musical Exercise at their home at their convenient time.

\subsection{Research Hypotheses}

Based on the design goals of Musical Exercise, we have posed the following hypotheses:

-Hypothesis 1: Musical Exercise will be rated as a usable exercise assistance system by the blindfolded participants. This question will be answered by the System Usability Scale (SUS) score.

-Hypotheses 2: Musical Exercise will help blindfolded participants do exercise as effectively as sighted participants do. This question will be answered based on the effects on consistency, completion time, and workload by comparing blindfolded group with audio conditions and sighted group without audio condition.

Musical Exercise has two distinct audio profiles. Given that the effects of different types of auditory displays vary depending on specific users, tasks, and contexts, we have more exploratory hypotheses about the differences of two audio profiles as follows.

-Hypotheses 3a: Continuous sound will assist the blindfolded participants to do the exercise equivalent to those in the visual without audio condition. This will be answered by comparing the effects of continuous sound on exercise.

-Hypotheses 3b: Discrete sound will assist the blindfolded participants to do the exercise equivalent to those in the visual without audio condition. This will also be answered by comparing the effects of discrete sound on exercise.

\subsection{Technical Details}

In Musical Exercise, participants performed two exercises squat and wall sit in front of a Microsoft Kinect device. Microsoft Kinect, which is a non-wearable device, can detect the participant's body movements and Musical Exercise used 
the Kinect data to recognize the specific exercise movement. Kinect was placed on a desk in front of the participant. The musical exercise application measured how many repetitions the participant completed, how consistent these movements were and how much time the participant took to complete the movement. Also, our application provided audio feedback so that the participant could understand the quality of the exercise such as how fast and when the participant completed the full range of motion for that specific exercise.

Musical Exercise was developed in C\# using Microsoft Kinect for Windows Software Development Kit (SDK) version 2.0. The Displaying body data template (source: http://kinect.github.io/tutorial/) was used to develop the application. This code template shows how to get BodyFrame from Kinect, using CoOrdinateMapper for $3 \mathrm{D}(\mathrm{x}, \mathrm{y}, \mathrm{z})$ positions of different body joints and displaying the human body as a skeleton using the XAML figure drawing. On top of this code template, we added our own algorithm for matching a specific exercise - either the squat or the wall sit exercise.

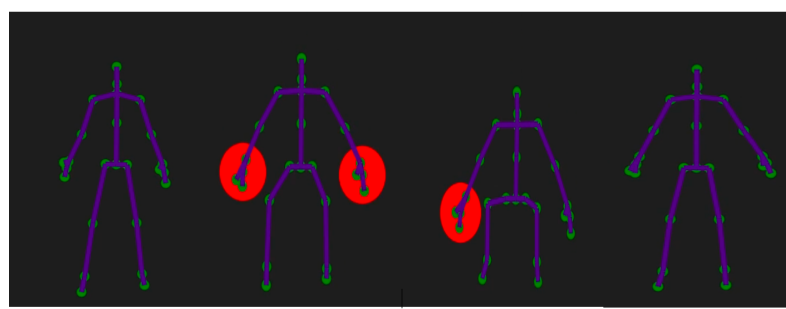

Figure 1: From left to right, movement of the exercise

To decide the requirements for a valid exercise repetition, we interviewed two experts (i.e., a physical exercise researcher and a professional competitor in exercise competitions). They both suggested squat and wall sit as a good full body exercise and recommended the full range of motion. If one's thighs are in parallel to the ground in squat or wall sit position, it is indicated as a good form of those exercises [15]. They also emphasized that the consistency in exercise movement is a crucial indicator of successful exercise. Following their advice, we implemented the exercise detection algorithm. Two knee joints and two hip points are detected and their coordinate positions are calculated. During each repetition of exercise, the time to complete the exercise is calculated and the lowest squat or wall sit with respect to the Kinect position is calculated.

The two exercises incorporate the movement which is from standing up to going down to the position when the knees are in straight line with the hip, which makes a 90 degree angle in the knee and has the thigh parallel to the ground. The exercise ends when going back to the standing position. The difference between squat and wall sit exercises is that in wall sit exercise, the user has to hold the sitting position longer and has back support of the wall. If the participant does not do the full range motion of an exercise, it is not detected as a successful exercise repetition.

While the participant is in exercise motion, one of the two audio profiles will be played based on the participant's state in the exercise motion. The continuous audio profile has eight notes. In the full range motion of an exercise, these eight notes were set to be played based on the participant's position or state in the exercise motion. These are MIDI notes ranging from MIDI number 60 to 71 . First note is played when the distance value crosses first cut-off point ( 28 unit, 1 unit $=1 \mathrm{~cm}$ ). Sequentially, the notes are played with decreasing polarity when the distance value crosses $22,16,10,7,4,2$ and less than 2 . When the participant stands up to complete the movement, the same eight notes are played in the reverse order. The range of motion is not big enough to accommodate eight MIDI notes sound separately with time gaps in between. Thus, this audio profile sounds like a continuous audio in practice. Hence, this audio profile is named continuous audio. If the participants are familiar with at what position which note is played, they can remember the note progression or sequence and try to mimic each time they do the exercise. In short, the sound in the continuous audio profile specifically produces feedback on if the participant is doing the exercise in a correct form. If the participant does not do the exercise in an ideal form, then the participant will hear different sound patterns compared to the sound of the ideal form of exercise.

The discrete audio profile was designed in a simplistic way with three MIDI notes. It has a starting note $(\mathrm{GH}-44)$ at the first cut-off points when the exercise begins, an intermediate note (E-64) when the participant is at an acceptable depth (i.e., when the distance between knee points and hip points is almost zero), and lastly an ending note (C-84) to mark the completion. All three MIDI notes sound separately and distinguishably while the participant doing the exercise. Hence, the name of this audio profile is discrete audio. We conducted an evaluation experiment comparing these two audio profiles with participants doing both exercises.

\section{EVALUATION}

\subsection{Participants}

Eighteen sighted participants (Mage $=20$ years, 19-22; $13 \mathrm{M}$, 5 F) were recruited using the Psychology Subject Recruitment System at a university. As a proof of concept, we conducted a preliminary study with blindfolded sighted participants before conducting any evaluation study with people with visual impairments. According to Moll and Sallnäs [35], if any constraint is implied on the blindfolded sighted person, it is hoped to be applicable for people with visual impairments. Kanwal et al. [34] also showed in their experiment of a navigation assistance system, the walking speed of the blindfolded participants were less than blind people. Therefore, a system designed for people with visual impairments like Musical Exercise is likely to impose more restraints in terms of performance on a blindfolded sighted person than a person with visual impairments. Yet we recognize the fact this experiment can be best evaluated by conducting with people with visual impairments. In the pre-questionnaire, all participants expressed their interest to do exercise for better well-being. However, only ten wanted to use technological assistance to do exercise. Eight participants have used fitness applications before. Seventeen participants have done exercise in various forms (e.g., yoga, bodyweight exercises, playing games, barbell or dumbbell exercise or cardio) in their lives. All participants, at least, heard about squat and wall sit exercise before. Eleven participants do squat exercise sometimes and 13 participants do wall sit exercise sometimes in their daily lives.

\subsection{Experimental Procedure and Conditions}

Once the participants signed the consent form and answered the pre-questionnaire, they were briefed in short about the exercise application. The participants were then assigned to the following six conditions in random order: Visual without audio condition, blindfolded without audio condition, visual with continuous audio condition, blindfolded with continuous audio condition, visual with discrete audio condition, and blindfolded with discrete audio condition. The experiment used the within-subjects design. In the visual conditions, 
participants saw the application running on a desktop monitor in front of them. The Kinect device was beside the monitor. The participants were asked to perform two exercises - squat and wall sit in random order. There were two sets of each exercise. In each set, the exercise needed to be repeated five times. There was 2-3 minute rest time between the sets. In the continuous audio conditions, the participants heard the continuous audio profile (eight MIDI notes) from the desktop monitor speaker while doing exercise. In the discrete audio conditions, the participants heard the discrete audio profile (three MIDI notes).

\section{RESULTS}

Firstly, we present how usable the Musical Exercise system is. Then, we report the effectiveness of Musical Exercise by discussing consistency of the exercise across different conditions. We also checked whether there is any effect of completion time among the conditions. After that, we show the workload measurement results across different experimental conditions. Note that our goal is to show that blindfolded group using Musical Exercise can do exercise as effectively as non-blindfolded group without any audio aid. Thus, we aim to show statistically not significant results between those pairs. Lastly, we present subjective questionnaire results.

\subsection{System Usability}

We used the System Usability Scale or SUS (source: https://www.usability.gov/how-to-and-tools/methods/systemusability-scale.html) to measure the usability of our system. SUS is a set of ten questions where each question has five answer options from "Strongly disagree" to "Strongly agree" scale. The score is calculated on a total of 100 points (for details about calculation, see herehttps://measuringu.com/sus/). SUS is a reliable and effective way to measure a system's usability [3]. After completing all the conditions, each participant answered the ten SUS questions. The mean SUS score for Musical Exercise was 71.53 out of total score 100 with a standard deviation of 8.45 calculated from 18 participants' data. The maximum SUS score was 85 and the minimum was 55 among 18 participants' SUS scores. Based on the results from 500 studies, the average SUS score 68 or above is considered an "above average" system (sources: https://measuringu.com/sus/ and https://www.usability.gov/how-to-and-tools/methods/systemusability-scale.html). Another study [3] reported that a SUS score of 71.4 with standard deviation of 11.6 can be described as a "good" system. Taken together, the Musical Exercise system stands as an "above average" or "good" usable system.

\subsection{Effectiveness of the System}

To measure the effectiveness of Musical Exercise, first we analyzed how consistently the participants did the exercise across the six conditions by calculating the variance data of distance values. Small variance means more consistency. However, consistency can also come from a bad form of exercise. For example, if the participants do the exercise consistently by maintaining a proper form almost all the time, then the variance of distance values will be low. In this case, this small variance would be good because the participants did the exercise constantly in the proper form. However, even though the participants consistently did the exercise, they might not sufficiently sit to the acceptable level as a successful position. Then, it would be consistently bad exercise. To distinguish these cases, we also analyzed whether consistency is good or bad by comparing mean distance values between knee and hip when the participants sit in both exercises across conditions. Figure 2 shows the distance between knee and hip joints.

Deciding good or bad consistency needs a base condition with which we can compare the conditions in question. We selected the visual without audio condition as the baseline condition because if sighted people do not use any exercise assistance system, they would do the exercise just like in the visual without audio condition in this experiment. Therefore, we focused on comparing the mean distance values of the blindfolded with audio conditions (continuous and discrete) with the mean distance values of the visual without audio condition after checking consistency of the conditions. Because of this reason, we directly conducted planned pairwise comparisons using paired samples t-tests, instead of conducting an overall ANOVA first and then using the Bonferroni correction. Therefore, we maintained a traditional alpha level (0.05) [33]. Each mean distance value represented the mean of five repetitions' distance values between the knee joints and the hip joints of a participant.

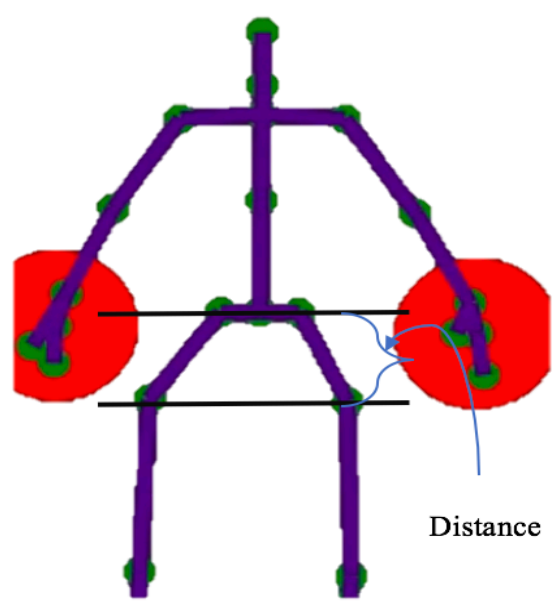

Figure 2: Distance between knee joints and hip joints

\subsubsection{Effects on consistency}

To measure the consistency of the exercise, we calculated the variance in distance between knee and hip positions when participants repeated the same exercise. This variance indicates how perfectly the participants did the exercise since equal or less than zero in the distance is considered a good form for both squat and wall sit exercises. Table 1 shows the variance data of squat exercise among six experimental conditions. From Table 1 , we can see that the variance values of the visual with discrete audio condition and the blindfolded with discrete audio condition are the least among the six conditions if we consider total sum of variance, mean of variance, or even variance of variance values.

The visual with continuous audio and the blindfolded with continuous audio conditions have the biggest variance values among the six conditions. With discrete audio either blindfolded or not, the participants seemed to perform squat more consistently than with the visual without audio condition or the blindfolded without audio condition. However, with continuous audio either blindfolded or not, the participants seemed to perform squat more inconsistently than the visual without audio condition or the blindfolded without audio condition. 


\begin{tabular}{|c|c|c|c|c|}
\hline Conditions & Sum & $\begin{array}{c}\text { Mean of } \\
\text { variance }\end{array}$ & $\begin{array}{c}\text { Variance of } \\
\text { variance }\end{array}$ & SD \\
\hline $\begin{array}{c}\text { Visual w/o } \\
\text { Audio }\end{array}$ & $\begin{array}{c}594 . \\
159\end{array}$ & 33.009 & 1322.933 & 36.372 \\
\hline $\begin{array}{c}\text { Blindfolded } \\
\text { w/o Audio }\end{array}$ & $\begin{array}{c}786 . \\
739\end{array}$ & 43.708 & 3017.291 & 54.929 \\
\hline $\begin{array}{c}\text { Visual w/ } \\
\text { continuous A }\end{array}$ & $\begin{array}{c}1228 \\
.879\end{array}$ & 68.271 & 15138.509 & 123.039 \\
\hline $\begin{array}{c}\text { Visual w/ } \\
\text { discrete A }\end{array}$ & $\begin{array}{c}445 . \\
287\end{array}$ & 24.738 & 837.089 & 28.932 \\
\hline $\begin{array}{c}\text { Blindfolded w/ } \\
\text { continuous A }\end{array}$ & $\begin{array}{c}1012 \\
.042\end{array}$ & 56.225 & 7581.276 & 87.071 \\
\hline $\begin{array}{c}\text { Blindfolded w/ } \\
\text { discrete A }\end{array}$ & $\begin{array}{c}590 . \\
074\end{array}$ & 32.782 & 718.998 & 26.814 \\
\hline
\end{tabular}

Table 1: Variance in distance between knee and hip for squat in six experimental conditions

\begin{tabular}{|c|c|c|c|c|}
\hline Conditions & Sum & Mean & Variance & SD \\
\hline $\begin{array}{c}\text { Visual w/o } \\
\text { Audio }\end{array}$ & -466.583 & -25.921 & 118.738 & 10.897 \\
\hline $\begin{array}{c}\text { Blindfolded } \\
\text { w/o Audio }\end{array}$ & -500.434 & -27.802 & 92.724 & 9.629 \\
\hline $\begin{array}{c}\text { Visual w/ } \\
\text { continuous A }\end{array}$ & -461.375 & -25.632 & 63.465 & 7.966 \\
\hline $\begin{array}{c}\text { Visual w/ } \\
\text { discrete A }\end{array}$ & -493.971 & -27.443 & 55.033 & 7.418 \\
\hline $\begin{array}{c}\text { Blindfolded w/ } \\
\text { continuous A }\end{array}$ & -499.871 & -27.771 & 109.025 & 10.441 \\
\hline $\begin{array}{c}\text { Blindfolded w/ } \\
\text { discrete A }\end{array}$ & -483.542 & -26.863 & 102.882 & 10.143 \\
\hline
\end{tabular}

Table 3: Mean data for squat exercise in six experimental conditions

For wall sit, results show different patterns (Table 2). The variance of the visual with discrete audio condition is the most similar to the visual without audio, but still higher. The variance of the blindfolded with discrete audio condition is much higher than the visual without audio. The blindfolded with both audio conditions show even higher variance compared to the blindfolded without audio condition. Again, the visual with continuous audio condition and the blindfolded with continuous audio condition show high variance.

In sum, in the visual with discrete audio condition, the participants seemed to perform relatively consistently for both exercises. When in the blindfolded with discrete audio condition the participants performed squat more consistently than either the visual without audio condition or the blindfolded without audio condition, but it is not the same case for wall sit exercise. Next, we show whether the consistent form is good or bad.

To determine if the participants performed in a consistently good form or bad form, we took the mean distance values of the exercise. Previously, while checking if the exercise is done consistently, we have found that in the visual with discrete audio condition, the participants performed more consistently. While the participants in the blindfolded with discrete audio condition performed, at least, as good as those in the visual without audio condition for squat exercise, this was not shown in the same way for wall sit exercise. Participants in the continuous audio conditions (either blindfolded or not) did not provide much consistent results in either of the exercises. Therefore, we focus on the paired samples t-tests between the blindfolded with discrete audio condition mean data and the visual without audio condition mean data.

\begin{tabular}{|c|c|c|c|c|}
\hline Conditions & Sum & $\begin{array}{c}\text { Mean of } \\
\text { variance }\end{array}$ & $\begin{array}{c}\text { Variance } \\
\text { of variance }\end{array}$ & SD \\
\hline $\begin{array}{c}\text { Visual w/o } \\
\text { Audio }\end{array}$ & $\begin{array}{c}457.7 \\
24\end{array}$ & 25.429 & 801.7 & 28.314 \\
\hline $\begin{array}{c}\text { Blindfolded } \\
\text { w/o Audio }\end{array}$ & $\begin{array}{c}923.2 \\
43\end{array}$ & 51.291 & 1996.086 & 44.678 \\
\hline $\begin{array}{c}\text { Visual w/ } \\
\text { continuous A }\end{array}$ & $\begin{array}{c}818.4 \\
46\end{array}$ & 45.469 & 4795.157 & 69.247 \\
\hline $\begin{array}{c}\text { Visual w/ } \\
\text { discrete A }\end{array}$ & $\begin{array}{c}517.5 \\
72\end{array}$ & 28.754 & 749.419 & 27.376 \\
\hline $\begin{array}{c}\text { Blindfolded w/ } \\
\text { continuous A }\end{array}$ & $\begin{array}{c}1001 . \\
747\end{array}$ & 55.653 & 7767.746 & 88.135 \\
\hline $\begin{array}{c}\text { Blindfolded w/ } \\
\text { discrete A }\end{array}$ & $\begin{array}{c}1026 . \\
737\end{array}$ & 57.041 & 5564.932 & 74.598 \\
\hline
\end{tabular}

Table 1: Variance in distance between knee and hip for wall sit in six experimental conditions

\begin{tabular}{|c|c|c|c|c|}
\hline Conditions & Sum & Mean & Variance & SD \\
\hline $\begin{array}{c}\text { Visual w/o } \\
\text { Audio }\end{array}$ & -498.673 & -27.704 & 52.048 & 7.214 \\
\hline $\begin{array}{c}\text { Blindfolded } \\
\text { w/o Audio }\end{array}$ & -454.719 & -25.262 & 45.291 & 6.729 \\
\hline $\begin{array}{c}\text { Visual w/ } \\
\text { continuous A }\end{array}$ & -492.174 & -27.343 & 98.564 & 9.928 \\
\hline $\begin{array}{c}\text { Visual w/ } \\
\text { discrete A }\end{array}$ & -491.446 & -27.303 & 52.291 & 7.231 \\
\hline $\begin{array}{c}\text { Blindfolded w/ } \\
\text { continuous A }\end{array}$ & -466.774 & -25.932 & 61.291 & 7.829 \\
\hline $\begin{array}{c}\text { Blindfolded w/ } \\
\text { discrete A }\end{array}$ & -437.104 & -24.284 & 154.838 & 12.443 \\
\hline
\end{tabular}

Table 4: Mean data for wall sit exercise in six experimental conditions

First for the squat exercise, Table 3 shows the mean values and standard deviation of mean distance data. Paired samples t-test between the blindfolded with discrete audio condition $(M=$ $26.863, S D=10.14)$ and the visual without audio condition $(M$ $=-25.92, S D=10.9$ ) shows no significant differences, $t(17)=$ $0.269, p=0.79$. Similarly, for the wall sit exercise, Table 4 shows the mean values and standard deviation of mean distance data. The paired samples t-test between the blindfolded with discrete audio $(M=-24.28, S D=12.44)$ and the visual without audio condition $(M=-27.7, \mathrm{SD}=7.2)$ shows no significant differences, $t(17)=-1.009, p=0.32$. Both of the t-test results suggest that when in the blindfolded with discrete audio condition participants performed not significantly different from the proper form of the exercise when compared to the visual without audio condition as a baseline.

In short, in the blindfolded with discrete audio condition, the participants consistently performed the proper form of squat as same as in the visual without audio condition and performed inconsistently but still as good form of wall sit as in the visual without audio condition.

\subsubsection{Effects on completion time}

Musical Exercise calculated the time from the beginning of an exercise to the end. This is the completion time of the exercise. We wanted to observe if there is any significant difference in time when the same exercise is done for different conditions. Specifically, we wanted to find out if there was any significant difference of completion time during the exercises between the visual without audio condition versus the blindfolded with continuous and discrete audio conditions respectively.

Paired samples t-test between the blindfolded with continuous audio $(M=1479.64, S D=473.79)$ and the visual without audio condition $(M=1344.19, S D=416.35)$ for the squat exercise completion time shows no significant differences, $t(17)=$ - 
0.91, $p=0.37$. Paired samples t-test between the blindfolded with discrete audio condition $(M=1620.19, S D=553.89)$ and the visual without audio condition $(M=1344.19, S D=416.35)$ for the squat exercise completion time also shows no significant differences, $t(17)=-1.69, p=0.10$.

The above two t-tests show that there is no significant difference between the visual without audio condition and the blindfolded with both continuous and discrete audio conditions in terms of squat exercise completion time.

Likewise, paired samples t-test between the blindfolded with continuous audio condition $(M=2070.22, S D=790.65)$ and the visual without audio condition $(M=2369.94, S D=$ 1216.49) for the wall sit exercise completion time shows no significant differences, $t(17)=0.88, p=0.39$. Paired samples t-test between the blindfolded with discrete audio condition ( $M$ $=2522.58, S D=1395.97)$ and the visual without audio condition $(M=2369.94, S D=1216.49)$ for the wall sit exercise completion time also shows no significant differences, $t(17)=$ $-0.35, p=0.73$. Therefore, whether it is squat or wall sit exercise, the exercise completion time is not significantly different in the blindfolded with one of two different audio (continuous and discrete) conditions than in the visual without audio condition. It means that while blindfolded with the aid of audios, the participant did not suffer from quickness or lag of completion time compared to a sighted person doing the exercise in the visual without audio condition.

\subsection{Workload Measure}

We used NASA Task Load Index or NASA-TLX for measuring the participants' perceived workload of Musical Exercise. After completing each of the six conditions, every participant answered the NASA-TLX questions. Based on their overall ratings of all the six experimental conditions, we conducted paired samples t-tests between the conditions. Table 5 shows the mean and standard deviation of all the six conditions.

\begin{tabular}{|c|c|c|c|}
\hline Conditions & Sum & Mean & SD \\
\hline $\begin{array}{c}\text { Visual w/o } \\
\text { Audio }\end{array}$ & 639.96 & 35.55 & 18.38 \\
\hline $\begin{array}{c}\text { Blindfolded w/o } \\
\text { Audio }\end{array}$ & 665.97 & 36.99 & 18.20 \\
\hline $\begin{array}{c}\text { Visual w/ } \\
\text { continuous A }\end{array}$ & 645.67 & 35.87 & 19.24 \\
\hline $\begin{array}{c}\text { Visual w/ } \\
\text { discrete A }\end{array}$ & 640.97 & 35.61 & 17.71 \\
\hline $\begin{array}{c}\text { Blindfolded w/ } \\
\text { continuous A }\end{array}$ & 708.65 & 39.37 & 19.24 \\
\hline $\begin{array}{c}\text { Blindfolded w/ } \\
\text { discrete A }\end{array}$ & 732.32 & 40.18 & 17.21 \\
\hline
\end{tabular}

Table 5: Overall rating score of NASA-TLX for six experimental conditions

Paired samples t-test between the blindfolded with continuous audio $(M=39.37, S D=19.24)$ and the visual without audio condition $(M=35.55, S D=18.38)$ shows no significant differences, $t(17)=-0.61, p=0.55$. Paired samples t-test between the blindfolded with discrete audio condition $(M=$ $40.18, S D=17.21)$ and the visual without audio condition $(M$ $=35.55, S D=18.38$ ) shows no significant differences, $t(17)=$ $-0.78, p=0.44$. These two t-tests show that there is no significant difference of overall workload between visual without audio condition and blindfolded with both continuous and discrete conditions in terms of perceived workload measure.

\subsection{Subjective Questionnaire}

After all experimental conditions were completed, participants answered the post-questionnaire: For "Which sound condition do you like better doing exercise?" with options of with audio or no audio, 14 participants $(77.78 \%)$ out of 18 participants chose the audio option (either continuous audio or discrete audio). For "Which exercise seemed difficult for you?" with options either wall sit or squat, 11 participants $(61.11 \%)$ out of 18 participants answered wall sit. Table 6 shows the positive results of other post questionnaire questions based on the Likert scale where 1 being strongly disagree and 5 being strongly agree.

\begin{tabular}{|c|c|c|}
\hline Questions & Mean & SD \\
\hline $\begin{array}{c}\text { "I think sound was source of information as } \\
\text { feedback for exercise" }\end{array}$ & 4.22 & 0.43 \\
\hline $\begin{array}{c}\text { "The exercise application would improve } \\
\text { your motivation to exercise." }\end{array}$ & 3.00 & 1.03 \\
\hline $\begin{array}{c}\text { "The exercise application's audio feedback } \\
\text { was intuitive" }\end{array}$ & 3.94 & 0.64 \\
\hline $\begin{array}{c}\text { "The exercise application's audio feedback } \\
\text { was useful." }\end{array}$ & 4.22 & 0.81 \\
\hline
\end{tabular}

Table 6: Subjective questionnaire scores

\section{DISCUSSION}

Musical Exercise is an exercise assistant application geared towards people with visual impairments. Our goal was to test if this system is usable and effective for people with visual impairments and if adding audio to the system works as an informative feedback medium. We conducted the experiment with sighted people being blindfolded. Though being blindfolded does not fulfill the premise of visual impairments, still, the experiment gives us insight on how people in general can respond to Musical Exercise. The SUS score of Musical Exercise showed that it is an "above average" or "good" system in terms of usability. For squat, the participants in the blindfolded with discrete audio condition tended to show more consistent (i.e., less variance) exercise than in the blindfolded without audio condition in terms of distance values. Also, the paired samples t-test showed that there was no significant difference between the blindfolded with discrete audio condition and the visual without audio condition. Therefore, with this discrete audio, Musical Exercise could improve consistency and assist the participants in maintaining a good form of squat exercise just as a sighted person would do in the visual without audio condition. However, for wall sit exercise, the results were different from the squat case. The performance of the participants was inconsistent both in the blindfolded with discrete audio condition and in the blindfolded with continuous audio condition. The reason seems to lie in participants being blindfolded and doing the wall sit exercise. Firstly, in wall sit exercise, the participants had back support which helped the participants do the exercise. In the blindfolded with audio conditions, the audio feedback and the physical contact to the wall may conflict with each other or even be combined into ambiguous feedback which might confuse the participants. Secondly, wall sit is a dependent exercise having the back-support component, whereas squat is an independent exercise. In squat, the participants developed their own rhythm with the audio feedback and followed it well, but in wall sit, the back support may have hampered the free movement of the participant which might contribute to the bad performance when in the blindfolded with audio conditions. Based on the results of the 
experiment, using discrete audio feedback can be recommended only for the squat exercise. Further research and validation is needed to improve audio profiles for wall sit. Nonetheless, in both exercises, audio played an important role by maintaining consistency or at least to ensure the proper form of the exercise. Another important factor is that the blindfolded with discrete audio condition has no significant difference with the visual without audio condition in terms of completion time and workload measure. In effect, people could do exercise in the blindfolded with discrete audio condition as good as the visual without audio condition. On a side note, results also showed that adding the discrete audio profile can also enhance performance of the sighted group compared to the sighted group without audio. However, not all audios were helpful. For both squat and wall sit exercises, in the blindfolded with continuous audio condition, the participants performed worse than in the blindfolded without audio condition. This result demonstrates that good audio design is crucial because it can assist people with visual impairments to perform exercises in a better way. In conclusion, our first two hypotheses are supported in terms of usability and effective exercise assistance system. Between the third (H3a) and fourth ( $\mathrm{H} 3 \mathrm{~b})$ hypotheses, only the third hypothesis (discrete audio) was partly supported by the results. Even though we were unable to conduct an evaluation study with people with visual impairments, based on the blindfolded conditions, Musical Exercise seems to be promising to be helpful for people with visual impairments. Also, a large portion of the participants $(77.78 \%)$ preferred to do the exercises with audio. They also commented that audio was intuitive and a source of information. Among the audio profiles, some participants liked the discrete audio profile, commenting that it helped "doing exercise in a correct form" or "ending repetition audio is good", etc. Some participants found that "continuous audio was hard to follow or even distracting". Only one participant who had music knowledge commented that the continuous audio profile was "more helpful in blindfolded conditions" and "could recognize a pattern in continuous audio". The evaluation of Musical Exercise shows that the participants prefer to do exercise with audio rather than no audio. More importantly, the participants thought that the audio feedback was helpful and informational. What distinguishes Musical Exercise from previous research studies is that the feedback system is intuitive and nonobtrusive using audio. Moreover, the use of non-speech sounds in Musical Exercise makes it a unique system as the non-speech sounds customize the feedback for the participants. Another feature of Musical Exercise is a nonwearable system which can be used at home environment with less human assistance.

Though the discrete audio profile was better perceived by our participants, at the same time it indicates that further iterative user evaluation is needed. Audio feedback seems to be essential to guide users with visual impairments to do the exercise. It helps them consistently maintain the proper form of exercise. However, it can be done only when the audio is properly designed. In future, more exercises can be included in the Musical Exercise platform. We can also add more features to the current exercises (e.g., the degree of inclination of the back). It will require researchers to seek more experts' opinions especially including iterative improvements of the system by evaluating and taking opinions of people with visual impairments on the design of the system. Also, Musical Exercise needs to be further evaluated by people with visual impairments. Overall, Musical Exercise has room for improvements.

\section{CONCLUSION}

Technology is continuously shaping our lives. Using technology in the area of solving accessibility and assistance issues for people seems to be promising. To use technology to its full potential, we need to create a usable and effective system. In the same line, the Musical Exercise platform aims to help people with visual impairments do exercise more independently. We designed the Musical Exercise system and tested it with two different types of audio feedback, compared to no audio condition. It seems that Musical Exercise is promising and heading to the right direction of being an effective and usable system for doing exercises not only for people with visual impairments, but also for sighted people. It not only worked as a feedback system but also was received positively so that it can also serve as a source of enjoyment during the exercises. Based on the results, we can make some design guidelines or recommendations for systems such as Musical Exercise. We hope that this system can play a major role in the field of using technology for people with disabilities and guide other researchers to design a more usable and effective technology.

\section{REFERENCES}

[1] Albiol-Pe' rez, S., Lozano-Quilis, J., Gil-Go'mez, H., GilGo'mez, J., and Llorens, R. Virtual rehabilitation system for people with parkinsons dis- ease. In 9th international conference on disability, virtual reality and associated technologies (ICDVRAT) (2012), pp. 423-427.

[2] Augestad, L. B., and Jiang, L. Physical activity, physical fitness, and body composition among children and young adults with visual impairments: A systematic review. British Journal of Visual Impairment 33, 3 (2015), 167-182.

[3] Bangor, A., Kortum, P., and Miller, J. Determining what individual sus scores mean: Adding an adjective rating scale. Journal of usability studies 4, 3 (2009), 114-123.

[4] Bolton, J., Lambert, M., Lirette, D., and Unsworth, B. Paperdude: A virtual reality cycling exergame. In CHI '14 Extended Abstracts on Human Factors in Computing Systems (New York, NY, USA, 2014), CHI EA '14, ACM, pp. 475478.

[5] Buttussi, F., Chittaro, L., and Nadalutti, D. Bringing mobile guides and fitness activities together: A solution based on an embodied virtual trainer. In Proceedings of the 8th Conference on Human-computer Interaction with Mobile Devices and Services (New York, NY, USA, 2006), MobileHCI '06, ACM, pp. 29-36.

[6] Conner, C., and Poor, G. M. Correcting exercise form using body track- ing. In Proceedings of the $2016 \mathrm{CHI}$ Conference Extended Abstracts on Human Factors in Computing Systems (New York, NY, USA, 2016), CHI EA '16, ACM, pp. 3028-3034.

[7] Elliott, D., Carr, S., and Orme, D. The effect of motivational music on sub- maximal exercise. European Journal of Sport Science 5, 2 (2005), 97-106.

[8] Fletcher, G. F., Balady, G., Blair, S. N., Blumenthal, J., Caspersen, C., Chaitman, B., Epstein, S., Froelicher, E. S. S., Froelicher, V. F., Pina, I. L., et al. Statement on exercise: benefits and recommendations for physical activity programs for all Americans. Circulation 94, 4 (1996), 857-862.

[9] Ganesan, S., and Anthony, L. Using the kinect to encourage older adults to exercise: A prototype. In CHI '12 Extended Abstracts on Human Factors in Computing Systems (NY, USA, 2012), CHI EA ’12, ACM, pp. 2297-2302. 
[10] Gerling, K. M., Kalyn, M. R., and Mandryk, R. L. Kinectwheels: Wheelchair- accessible motion-based game interaction. In CHI '13 Extended Abstracts on Human Factors in Computing Systems (New York, NY, USA, 2013), CHI EA '13, ACM, pp. 3055-3058.

[11] Gowin, M., Cheney, M., Gwin, S., and Wann, T. F. Health and fitness app use in college students: A qualitative study. American Journal of Health Education 46, 4 (2015), 223-230. [12] Houwen, S., Hartman, E., and Visscher, C. Physical activity and motor skills in children with and without visual impairments. Medicine and science in sports and exercise 41, 1 (2009), 103-109.

[13] Huang, M.-C., Chen, E., Xu, W., and Sarrafzadeh, M. Gaming for up- per extremities rehabilitation. In Proceedings of the $2 \mathrm{Nd}$ Conference on Wireless Health (New York, NY, USA, 2011), WH '11, ACM, pp. 27:1-27:2.

[14] Jacko, J. A., Scott, I. U., Sainfort, F., Barnard, L., Edwards, P. J., Emery, V. K., Kongnakorn, T., Moloney, K. P., and Zorich, B. S. Older adults and visual impairment: What do exposure times and accuracy tell us about performance gains associated with multimodal feedback? In Proceedings of the SIGCHI Conference on Human Factors in Computing Systems (NY, USA, 2003), CHI '03, ACM, pp. 33-40.

[15] Kasim, P. Optimizing squat technique. Strength and Conditioning Journal 29, 6 (2007), 10.

[16] Laukka, P., and Quick, L. Emotional and motivational uses of music in sports and exercise: A questionnaire study among athletes. Psychology of Music 41, 2 (2013), 198-215.

[17] Lin, T.-Y., Hsieh, C.-H., and Lee, J.-D. A kinect-based system for phys- ical rehabilitation: Utilizing tai chi exercises to improve movement disorders in patients with balance ability. In Proceedings of the 2013 7th Asia Modelling Symposium (Washington, DC, USA, 2013), AMS '13, IEEE Computer Society, pp. 149-153.

[18] Middelweerd, A., Mollee, J. S., van der Wal, C. N., Brug, J., and te Velde, S. J. Apps to promote physical activity among adults: a review and content analysis. International Journal of Behavioral Nutrition and Physical Activity 11, 1 (2014), 1-9. [19] Morelli, T., Foley, J., Columna, L., Lieberman, L., and Folmer, E. Vi-tennis: A vibrotactile/audio exergame for players who are visually impaired. In Proceedings of the Fifth International Conference on the Foundations of Digital Games (New York, NY, USA, 2010), FDG '10, ACM, pp. 147-154. [20] Muehlbradt, A., Koushik, V., and Kane, S. K. Goby: A wearable swimming aid for blind athletes. In Proceedings of the 19th International ACM SIGACCESS Conference on Computers and Accessibility (New York, NY, USA, 2017), ASSETS '17, ACM, pp. 377-378.

[21] Newbold, J. W., Bianchi-Berthouze, N., Gold, N. E., Tajadura- Jime nez, A., and Williams, A. C. Musically informed sonification for chronic pain rehabilitation: Facilitating progress \&\#38; avoiding over-doing. In Proceedings of the $2016 \mathrm{CHI}$ Conference on Human Factors in Computing Systems (New York, NY, USA, 2016), CHI '16, ACM, pp. 5698-5703.

[22] Palacios-Navarro, G., Garc' 1a-Magarin o, I., and RamosLorente, P. A kinect- based system for lower limb rehabilitation in parkinson's disease patients: a pilot study. Journal of Medical Systems 39, 9 (2015), 103.
[23] Rector, K., Bennett, C. L., and Kientz, J. A. Eyes-free yoga: An exergame using depth cameras for blind \&\#38; low vision exercise. In Proceedings of the 15th International ACM SIGACCESS Conference on Computers and Accessibility (New York, NY, USA, 2013), ASSETS '13, ACM, pp. 12:112:8.

[24] Salmon, P. Effects of physical exercise on anxiety, depression, and sensitivity to stress: a unifying theory. Clinical psychology review 21, 1 (2001), 33-61.

[25] Shapiro, D. R., Moffett, A., Lieberman, L., and Dummer, G. M. Perceived competence of children with visual impairments. Journal of Visual Impairment \& Blindness 99, 1 (2005), 15.

[26] Singh, A., Klapper, A., Jia, J., Fidalgo, A., TajaduraJime nez, A., Kanakam, N., Bianchi-Berthouze, N., and Williams, A. Motivating people with chronic pain to do physical activity: Opportunities for technology design. In Proceedings of the SIGCHI Conference on Human Factors in Computing Systems (New York, NY, USA, 2014), CHI '14, ACM, pp. 2803-2812.

[27] Taylor, D. Physical activity is medicine for older adults. Postgraduate Medical Journal 90, 1059 (2014), 26-32.

[28] Wang, Q., Turaga, P., Coleman, G., and Ingalls, T. Somatech: An exploratory interface for altering movement habits. In Proceedings of the Extended Abstracts of the 32Nd Annual ACM Conference on Human Factors in Computing Systems (NY, USA, 2014), CHI EA'14, pp. 1765-1770.

[29] Warburton, D. E., Nicol, C. W., and Bredin, S. S. Health benefits of physical activity: the evidence. Canadian Medical Association Journal 174, 6 (2006), 801-809.

[30] Weil, E., Wachterman, M., McCarthy, E. P., Davis, R. B., O'day, B., Iezzoni, L. I., and Wee, C. C. Obesity among adults with disabling conditions. Jama 288, 10 (2002), 1265-1268.

[31] Winoto, P., and Tang, T. Y. Sensory substitution to enable the visually im- paired to play an affordable wearable mobile game. In Adjunct Proceedings of the 2015 ACM International Joint Conference on Pervasive and Ubiquitous Com- puting and Proceedings of the 2015 ACM International Symposium on Wearable Computers (New York, NY, USA, 2015), UbiComp/ISWC'15 Adjunct, ACM, pp. 193-196.

[32] Zayer, M. A., Tregillus, S., Bhandari, J., Feil-Seifer, D., and Folmer, E. Exploring the use of a drone to guide blind runners. In International ACM SIGACCESS Conference on Computers and Accessibility (Reno, NV, October 2016), pp. 263-264.

[33] Keppel, G., and Wicken, T. D. Design and analysis: A researcher's handbook. London, UK: Pearson Prentice Hall. (2004).

[34] Nadia Kanwal, Erkan Bostanci, Keith Currie, and Adrian F. Clark, "A Navigation System for the Visually Impaired: A Fusion of Vision and Depth Sensor," Applied Bionics and Biomechanics, vol. 2015, Article ID 479857, 16 pages, 2015. doi: $10.1155 / 2015 / 479857$

[35] Moll, Jonas, and Eva-Lotta Sallnäs. "Communicative functions of haptic feedback." In International Conference on Haptic and Audio Interaction Design, pp. 1-10. Springer, Berlin, Heidelberg, 2009. 